Check for updates

Cite this: RSC Adv., 2019, 9, 37484

Received 24th September 2019 Accepted 4th November 2019

DOI: 10.1039/c9ra07701d

rsc.li/rsc-advances

\section{Direct and sensitive detection of sulfide ions based on one-step synthesis of ionic liquid functionalized fluorescent carbon nanoribbons $\uparrow$}

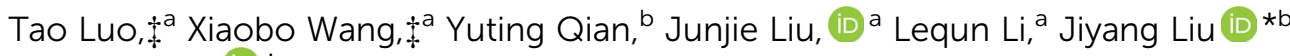 \\ and Jie Chen (D) *a
}

\begin{abstract}
Despite widely reported fluorescence sensors for cations, direct detection of anions is nevertheless still rare. In this work, ionic liquid-functionalized fluorescent carbon nanoribbons (IL-CNRs) are one-step synthesized and serve as the fluorescent probes for direct and sensitive detection of sulfide ions $\left(S^{2-}\right)$. The IL-CNRs are synthesized based on electrochemical exfoliation of graphite rods in a water-IL biphasic system. The as-prepared IL-CNRs exhibit uniform structure, high crystallinity, strong blue fluorescence (absolute photoluminescence quantum yield of $11.4 \%$ ), and unique selectivity towards $\mathrm{S}^{2-}$. Based on the fluorescence quenching of IL-CNRs by $\mathrm{S}^{2-}$, a fluorescence sensor is developed for direct, rapid and sensitive detection of $\mathrm{S}^{2-}$ in the range of $100 \mathrm{nM}$ to $1 \mu \mathrm{M}$ and $1-300 \mu \mathrm{M}$ with a low detection limit (LOD, $85 \mathrm{nM}$ ). Moreover, detection of $\mathrm{S}^{2-}$ in a real sample (tap water) is also demonstrated.
\end{abstract}

\section{Introduction}

Inorganic anions play a crucial role in industrial, biological and environmental fields. ${ }^{1}$ For instance, the sulfide $\left(\mathrm{S}^{2-}\right)$ ion is highly toxic and harmful to human health and the ecological environment. ${ }^{2-4}$ Usually, the $\mathrm{S}^{2-}$ ion is frequently distributed in natural water and wastewater samples because it is widely used in industrial processes (e.g. sulfur and sulfuric acid production, petroleum refineries, paper and pulp manufacturing, and sewage processing industries) and is also produced in biological systems (e.g. via reduction of sulfate ions by microbes or anaerobic processes of S-containing enzymes)..$^{4-6}$ As the $\mathrm{S}^{2-}$ ion can stimulate the mucosa and has an extreme effect on the nervous system, exposure to $\mathrm{S}^{2-}$ ions leads to unconsciousness and respiratory paralysis and is closely related to various diseases including Alzheimer's disease, Down's syndrome and cirrhosis of the liver. ${ }^{7-10}$ Therefore, simple, rapid and sensitive detection of $\mathrm{S}^{2-}$ is of great importance.

In comparison with other technologies including gas chromatography, ion chromatography, titrimetry, colorimetry, and electrochemical method, fluorescent sensors are particularly attractive because of high sensitivity, simple operation and rapid detection. ${ }^{3,11-17}$ Recently, fluorescent sensors based on

\footnotetext{
${ }^{a}$ Affiliated Tumor Hospital of Guangxi Medical University, 71 Hedi Road, Nanning 530021, PR China. E-mail: jiechen185@163.com

${ }^{b}$ Department of Chemistry, Zhejiang Sci-Tech University, 928 Second Avenue, Xiasha Higher Education Zone, Hangzhou, 310018, PR China. E-mail: liujy@zstu.edu.cn

$\dagger$ Electronic supplementary information (ESI) available. See DOI: 10.1039/c9ra07701d

¥ The two authors contributed equally.
}

different fluorescent probes have been widely reported for detection of cations. ${ }^{18,19}$ However, only few researches focus on indirect detection of anions using fluorescent off-on strategy. For instance, $\mathrm{Na}$ et al. reported that $\mathrm{Cu}^{2+}$ ions caused aggregation of the GQDs and thereby quenched fluorescence. ${ }^{12}$ As the GQDs- $\mathrm{Cu}^{2+}$ aggregates can be dissociated by adding $\mathrm{S}^{2-}$ ions and result in fluorescence turn-on, fluorescent detection of $\mathrm{S}^{2-}$ ions could be realized. Based on the same off-on strategies, graphitic carbon nitride quantum dots- $\mathrm{Hg}^{2+}, 7$ nitrogen and sulfur co-doped carbon dots (CDs)- $\mathrm{Hg}^{2+},{ }^{2}$ CDs-gold nanoparticles,${ }^{10}$ silver nanoparticles capped with $\mathrm{CDs},{ }^{9} \mathrm{CDs}^{-\mathrm{MnO}_{2}}$ nanosheets, ${ }^{13}$ quinoline- $\mathrm{Zn}^{2+},{ }^{2}$ calix $[4]$ arene- $\mathrm{Cu}^{2+},{ }^{11}$ and organic semiconductor polymer nanodots-Cu ${ }^{2+}$ systems $^{8}$ were also applied for the detection of $\mathrm{S}^{2-}$ ions. However, it still remains great challenge for improving the chemical selectivity of fluorescent probe to realize the direct detection of $\mathrm{S}^{2-}$.

Carbon-based fluorescent nanomaterials have attracted considerable interests as probes in fluorescent sensing due to good solubility, highly tunable photoluminescence properties and biocompatibility. ${ }^{20-24}$ Carbon nanoribbons (CNRs), which is thin elongated strips of $\mathrm{sp}^{2}$ bonded carbon atoms, have emerged as an exciting one-dimensional nanomaterial because of their large length-to-width ratio, abundant edge sides, high effective surface area, good flexibility, high conductivity, and tunable fluorescence. ${ }^{25-28}$ Due to the large interfacial area for $\pi-$ $\pi$ stacking in basal planes, CNRs exhibit potentials for the synthesis of advanced functional materials through simple modification or functionalization.

Ionic liquids (ILs) have been widely used in many fields because of a series of excellent physical and chemical properties. $^{21,28,29}$ Particularly, ILs can easily combine with carbon 
nanomaterials due to $\pi-\pi$ or cation $-\pi$ interactions and the obtained composite materials exhibit unique characteristics. $^{21,28-31}$ For instance, Wang et al. prepared highly charged CDs through one-pot pyrolysis with citric acid as carbon source and ionic liquid (1-aminopropyl-3-methylimidazolium bromide, [APMIm][Br]) as capping agent. ${ }^{32}$ Those IL-modified CDs exhibit capability of anion exchange and are able to act as fluorescent probe for direct detection of $\mathrm{Fe}(\mathrm{CN})_{6}{ }^{3-}$ anion. Our group also prepared IL-GQDs nanomaterial through postmodification of hydroxyl-functionalized GQDs (OH-GQDs) with 1-butyl-3-methyl imidazolium tetrafluoroborate $\left(\mathrm{BMIMBF}_{4}\right)$ under ultrasonic treatment. The as-prepared ILGQDs composite also displayed sensitive response towards $\mathrm{Fe}(\mathrm{CN})_{6}{ }^{3-}$, owing to the anion exchange ability of $\mathrm{IL} .{ }^{33}$ Inspired by these evidences, IL modified fluorescent nanocarbon materials possess great potentials for the direct detection of anions.

In this work, in contrast to anion detection based on fluorescence off-on mode, a new type of ionic liquid-functionalized fluorescent carbon nanoribbons (IL-CNRs) were prepared using one-step electrochemical method, which is able to achieve direct and sensitive detection of sulfide ions. The IL-CNRs were in situ synthesized based on electrochemical exfoliation of graphite rods in water-IL (1-butyl-3-methylimidazolium hexafluorophosphate, $[\mathrm{BMIM}] \mathrm{PF}_{6}$ ) biphasic system. The obtained ILCNRs exhibit bright fluorescence, high crystallinity and uniform structure. Based on the selective fluorescent quenching by $\mathrm{S}^{2-}$, IL-CNRs can be served as fluorescent probes for direct and sensitive detection of $\mathrm{S}^{2-}$. In comparison with the previous cation-based fluorescent sensor, we for the first time demonstrate the application of IL-CNRs for direct and selective detection of $\mathrm{S}^{2-}$.

\section{Experimental}

\subsection{Materials and reagents}

High-purity graphite rods were purchased from China National Medicines Co. Ltd (China). 1-Butyl-3-methylimidazolium hexafluorophosphate ([BMIM] $\left.\mathrm{PF}_{6}\right)$ (>99\%), ascorbic acid (AA), and 4(2-hydroxyethyl)-1-piperazineethanesulfonic acid (HEPES), $\mathrm{Na}_{2} \mathrm{~S}$ were purchased from Sigma Aldrich. Aqueous solutions of $\mathrm{Na}^{+}$, $\mathrm{K}^{+}, \mathrm{Zn}^{2+}, \mathrm{Mg}^{2+}, \mathrm{Fe}^{3+}, \mathrm{Ca}^{2+}, \mathrm{Al}^{3+}, \mathrm{Ni}^{2+}, \mathrm{Cu}^{2+}, \mathrm{Cd}^{2+}$ were prepared from their chloride salts. Solutions of $\mathrm{Pb}^{2+}, \mathrm{Ag}^{+}$and $\mathrm{Hg}^{2+}$ were prepared from their nitrate salts. Aqueous solutions of $\mathrm{CH}_{3}$ $\mathrm{COO}^{-}, \mathrm{HCO}_{3}{ }^{-}, \mathrm{H}_{2} \mathrm{PO}_{4}{ }^{-}, \mathrm{HPO}_{4}{ }^{2-}, \mathrm{NO}_{2}{ }^{-}, \mathrm{S}^{2-}, \mathrm{S}_{2} \mathrm{O}_{3}{ }^{2-}$ were prepared from their sodium salts. $\mathrm{SCN}^{-}, \mathrm{Cl}^{-}, \mathrm{NO}_{3}{ }^{-}, \mathrm{Br}^{-}$were prepared from their potassium salts. All chemicals were of analytical grade and used without further treatment. Aqueous solutions were prepared with ultrapure water $(18.2 \mathrm{M} \Omega \mathrm{cm}$, Milli-Q, Millipore).

\subsection{Characterizations}

Transmission electron microscopy (TEM) micrographs were obtained using a JEM-2100F transmission electron microscope (JEOL Ltd, Japan) at accelerating voltage of $200 \mathrm{kV}$. X-ray photoelectron spectroscopy (XPS) measurements were carried out on a PHI5300 at operating voltage of $14 \mathrm{kV}$ (PE Ltd., USA).
The Fourier transform infrared spectra (FTIR) were conducted with a Nicolet 5700 FTIR spectrometer (Thermo Electron Scientific Instruments Corp., USA). Fluorescence spectra were measured using FL 3C-11 spectrofluorometer (Horiba Scientific, USA).

\subsection{One-step electrochemical synthesis of IL-CNRs}

The IL-CNRs were one-step synthesized by electrochemical exfoliation of graphite rods. A two electrodes system was adopted with graphite rods $(d=3 \mathrm{~mm})$ as both anode and cathode. The electrolyte was water-IL ([BMIm] $\left.\left[\mathrm{PF}_{6}\right]\right)$ biphasic system (V/V, $4: 6)$. Graphite rods were vertically inserted into electrolyte solution at a distance of $3 \mathrm{~cm} .{ }^{21}$ A static voltage of $8 \mathrm{~V}$ was applied on graphite rod for $4 \mathrm{~h}$ with stirring the electrolyte. Afterwards, the resultant solution was centrifuged at $12000 \mathrm{rpm}$ for $10 \mathrm{~min}$ to remove large particles. To acquire ILCNRs, the supernatant solution was then filtered using a 0.22 $\mu \mathrm{m}$ membrane and dialyzed in a dialysis bag (with cut-off molecular weight of $1000 \mathrm{Da}$ ), which is followed by freezedrying.

\subsection{Fluorescent detection of $\mathrm{S}^{2-}$}

The selectivity of IL-CNRs towards different anions and cations were evaluated by measuring the fluorescent intensity of ILCNRs solution in presence of different cations $\left(\mathrm{Na}^{+}, \mathrm{K}^{+}, \mathrm{Zn}^{2+}\right.$, $\left.\mathrm{Mg}^{2+}, \mathrm{Fe}^{3+}, \mathrm{Ca}^{2+}, \mathrm{Al}^{3+}, \mathrm{Ni}^{2+}, \mathrm{Cu}^{2+}, \mathrm{Cd}^{2+}\right)$ or anions $\left(\mathrm{CH}_{3} \mathrm{COO}^{-}\right.$, $\mathrm{HCO}_{3}{ }^{-}, \mathrm{H}_{2} \mathrm{PO}_{4}{ }^{-}, \mathrm{SCN}^{-}, \mathrm{HPO}_{4}{ }^{2-}, \mathrm{S}^{2-}, \mathrm{Cl}^{-}, \mathrm{NO}_{3}{ }^{-}, \mathrm{NO}_{2}{ }^{-}, \mathrm{Br}^{-}$, $\left.\mathrm{S}_{2} \mathrm{O}_{3}{ }^{2-}\right)$.

For $\mathrm{S}^{2-}$ detection, standard stock solutions of $\mathrm{S}^{2-}(0.1 \mathrm{M})$ were prepared and further diluted stepwise to obtain different concentrations. HEPES buffer $(0.1 \mathrm{M}, \mathrm{pH} 7.0)$ containing AA (25 $\mu \mathrm{M})$ was applied as the buffer solution. After reaction of ILCNRs with different amount of $\mathrm{S}^{2-}$ for $1 \mathrm{~min}$ at room temperature, the fluorescent intensity of the solution was recorded with the excitation wavelength fixed at $340 \mathrm{~nm}$.

The relative fluorescence ratio $\left(F / F_{0}\right)$ or relative fluorescence quenching ratio $\left(F_{0}-F / F_{0}\right)$ were used to evaluate the quenching of IL-CNRs caused by ion, where $F_{0}$ and $F$ represent the fluorescent intensity of IL-CNRs solution in the absence and presence of ion, respectively.

\section{Results and discussion}

\subsection{Preparation and structure characterizations of IL-CNRs}

Electrochemical synthesis has particularly attracted attention owning to the inexpensive apparatus and easy operation under

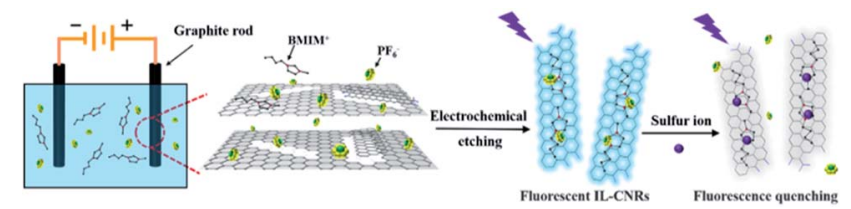

Fig. 1 Schematic illustration for the preparation of IL-CNRs and detection of sulfide ions. 
ambient conditions. ${ }^{34-36}$ As illustrated in Fig. 1, ionic liquidfunctionalized fluorescent carbon nanoribbons (IL-CNRs) were easily synthesized through one-step electrochemical exfoliation of graphite rods in water-IL (1-butyl-3-methylimidazolium hexafluorophosphate, $[\mathrm{BMIM}] \mathrm{PF}_{6}$ ) biphasic system. Owing to high ionic conductivity and wide electrochemical potential window, ILs shows potential as electrolyte in electrochemical synthesis. ${ }^{17,24}$ In the process of electrochemical exfoliation, the colour of electrolyte turned from colourless to pale yellow and afterward turned dark brown due to rapid flaking-off of abundant IL-CNRs. In comparison with the same procedure in pure water, this phenomenon cannot be observed, indicating the importance of ILs for improved conductivity and facilitated exfoliation. The formation of IL-CNRs can be observed by transmission electron microscopy (TEM) (Fig. 2). The length and width of IL-CNRs were about $40 \mathrm{~nm}$ and $5 \mathrm{~nm}$ (Fig. 2a-c), respectively. Clear lattice lines can be seen from the highresolution TEM (HRTEM) image (Fig. 2d), indicating good crystallinity. The lattice spaces are $0.26 \mathrm{~nm}$ and $0.38 \mathrm{~nm}$, that are consistent with the (100) and (002) lattice of graphite, respectively. ${ }^{21}$

The chemical composition of the as-prepared IL-CNRs were characterized by X-ray photoelectron spectroscopy (XPS). As shown in Fig. 3a, the XPS survey spectrum of IL-CNRs contains five distinct peaks of C 1s, N 1s, O 1s, P 2p, F 1s. The appearance of $\mathrm{N}, \mathrm{P}, \mathrm{F}$ reveals the formation of IL-CNRs nanocomposites. The $\mathrm{C}-\mathrm{C} / \mathrm{C}=\mathrm{C}\left(\mathrm{sp}^{2} \mathrm{C}\right)$ peak is prominent in high resolution spectrum of $\mathrm{C} 1 \mathrm{~s}$, suggesting the graphitic structure in CNRs (Fig. 3b). This result is consistent with the good crystallinity demonstrated in HRTEM image (Fig. 2d). The appearance of $\mathrm{C}-\mathrm{N}$ in high resolution spectrum of $\mathrm{C} 1 \mathrm{~s}$ (Fig. $3 \mathrm{~b}$ ), $\mathrm{N}-\mathrm{C}=\mathrm{C}$ and quaternary $\mathrm{N}$ in high resolution spectrum of $\mathrm{N}$ 1s (Fig. 3c) confirm the existence of $\mathrm{BMIM}^{+}$in IL-CNRs. The appearance of O 1s reveals the abundant oxygen-containing groups (Fig. 3d), suggesting good solubility of the as-prepared IL-CNRs. The F 1s peak (Fig. 3e) and P 2p peak (Fig. 3f) also prove the presence of

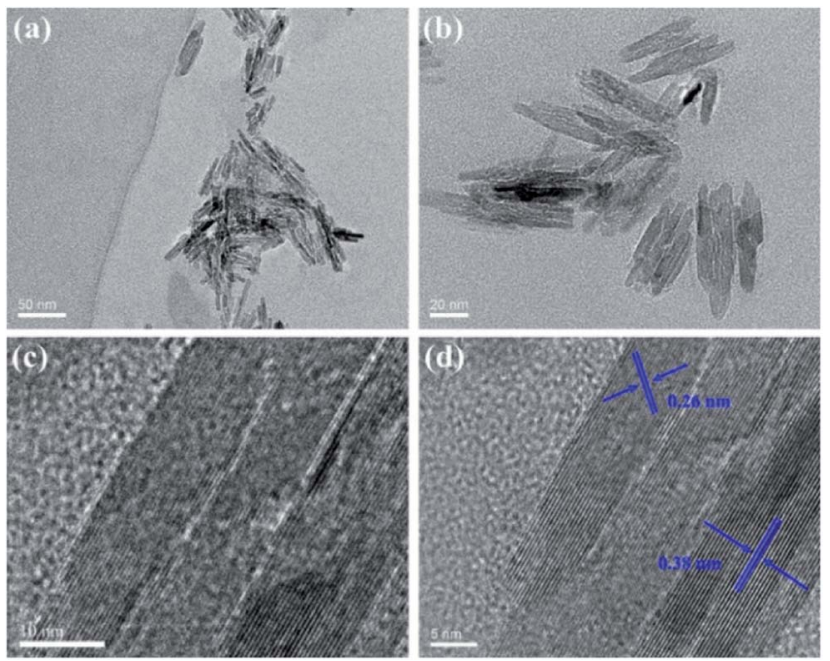

Fig. 2 TEM (a-c) and HRTEM (d) images of IL-CNRs at different magnification. The indicated data are lattice parameters of IL-CNRs.
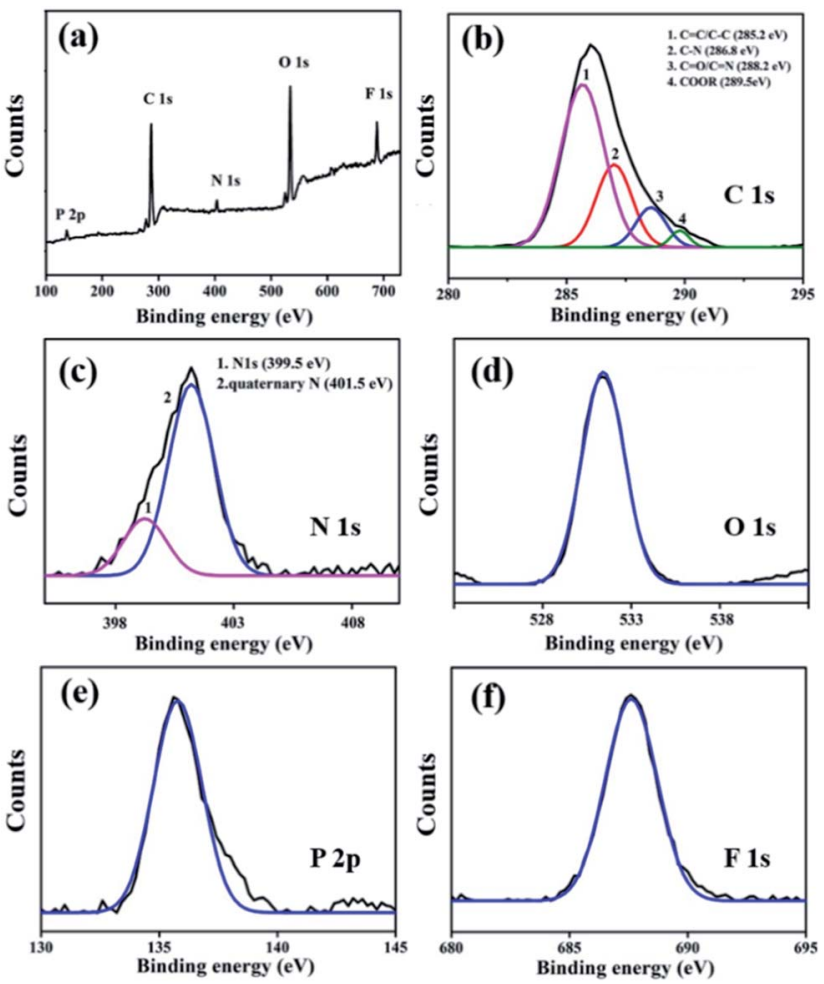

Fig. 3 XPS survey spectrum (a) and high resolution C1s (b), N1s (c), O1s (d), P2p (e) and F1s (f) spectra of IL-CNRs.

$\mathrm{PF}_{6}{ }^{-}$anion in IL-CNRs, indicating the hybridization between CNRs and IL. These results evidence the successful formation of $[\mathrm{BMIM}] \mathrm{PF}_{6}$ modified CNRs in the one-step electrochemical exfoliation of graphite. Consistently, Fourier transform infrared spectrum (FTIR) of IL-CNRs (Fig. S1 in ESI $\dagger$ ) also shows characteristic vibrations of $\mathrm{C}-\mathrm{H}\left(2962,2922\right.$, and $\left.2857 \mathrm{~cm}^{-1}\right), \mathrm{C}=\mathrm{C}$ and $\mathrm{C}=\mathrm{N}\left(1552 \mathrm{~cm}^{-1}\right),-\mathrm{OH}\left(3457 \mathrm{~cm}^{-1}\right), \mathrm{C}=\mathrm{O}\left(1640 \mathrm{~cm}^{-1}\right)$, and $\mathrm{PF}_{6}{ }^{-}$(1578 and $\left.1027 \mathrm{~cm}^{-1}\right)$, that agree well with [BMIM] $\mathrm{PF}_{6}$. In comparison with IL, UV-Vis spectrum of IL-CNRs exhibits the same large peak of $\pi-\pi^{*}$ transition $(\sim 240 \mathrm{~nm})$ and new peak at about $326 \mathrm{~nm}$ that corresponds to $\mathrm{n}-\pi^{*}$ transition (Fig. S2 in $\mathrm{ESI} \dagger)$. Taken together, $[\mathrm{BMIM}] \mathrm{PF}_{6}$ are proven to be composited with CNRs.

According to the above results, we propose that the mechanism for the preparation of IL-CNRs includes oxidative cleavage, ionic liquid intercalation and hybridization (Fig. 1). ${ }^{21,28-31}$ Firstly, the oxidation of water is expected to produce radicals (e.g. hydroxyl and oxygen radicals) when a high oxidation voltage is applied. Secondly, the oxidation of the edge planes opens up the edge sheets and leads to the expansion of the graphite anode, facilitating intercalation by $\mathrm{PF}_{6}{ }^{-}$between the graphene layers. Thirdly, the oxidative cleavage of the expanded graphene sheets generates CNRs. Finally, interaction between CNRs and $\mathrm{BMIM}^{+}$via $\pi-\pi$ or cation $-\pi$ interactions leads to stable IL-CNRs nanocomposites. In comparison with the preparation of CNRs using electron or ion beam etching, chemical ultrasound, lithographic patterning, oxidative cleavage, and chemical vapor deposition (CVD), ${ }^{37-40}$ this 
electrochemical method is simple, green and possesses potential for scalable production owing to inexpensive apparatus and easy operation.

\subsection{Fluorescence properties of IL-CNRs}

The fluorescence properties of IL-CNRs were investigated and results were demonstrated in Fig. 4. The IL-CNRs show good solubility in water and appears light-yellow under daylight, while exhibit strong blue luminescence under UV light (365 nm) (inset in Fig. 4b). The maximum emission is located at $430 \mathrm{~nm}$ and the maximum excitation wavelength is $340 \mathrm{~nm}$ (Fig. 4a). Like most luminescent carbon nanomaterials, the IL-CNRs also exhibit excitation-dependent fluorescence behavior (Fig. 4b). ILCNRs prepared under the same conditions independently exhibit the same maximus emission wavelength and a relative standard deviation (RSD) of $2.9 \%$ in fluorescence intensity (at $430 \mathrm{~nm}$ ), indicating high stability and repeatability of the preparation. As the excitation wavelength increases, the fluorescence emission slightly red-shifts, which could be ascribed to size heterogeneity and distribution of different emissive sites on the carbon ribbons. Absolute photoluminescence quantum yield of IL-CNRs is measured to be $11.4 \%$, which may be ascribed to the high crystallinity of IL-CNRs and the presence of $\mathrm{BMIM}^{+}$on IL-CNRs surface which has electron-withdrawing nitrogen groups. The lifetime of B-GQDs is 5.9 ns (Fig. S3 in ESI $\dagger$ ). The IL-CNRs also exhibits good photostability. As shown in Fig. S4 (ESI $\dagger$ ), even after being illuminated for $4 \mathrm{~h}$ under UV light $(365 \mathrm{~nm})$, the fluorescent intensity remains 91\%, indicating good anti-photobleaching property.

\subsection{Ion selectivity of IL-CNRs}

Ionic liquids modified carbon materials have proven to exhibit anion exchange ability and have potential in direct detection of anion. Thus, the selectivity of the as-prepared IL-CNRs towards different anions were investigated. As revealed in Fig. 5a, the fluorescence of IL-CNRs is strongly quenched by $\mathrm{S}^{2-}$, but not the other physiologically or environmentally relevant anions (Fig. 5a). The lifetime of IL-CNRs in presence $\mathrm{S}^{2-}$ is also revealed to be 5.9 ns (Fig. S5 in ESI $\dagger$ ). The unchanged fluorescence lifetime of IL-CNRs in the presence of $\mathrm{S}^{2-}$ indicates no charge transfer and exciton recombination process. ${ }^{18}$ The quenching
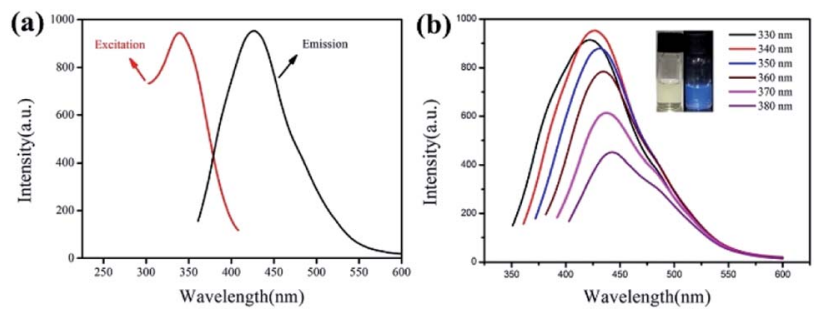

Fig. 4 (a) The FL excitation spectrum of IL-CNRs obtained at the emission wavelength of $430 \mathrm{~nm}$ and FL emission spectrum of IL-CNRs obtained with the excitation wavelength of $340 \mathrm{~nm}$. (b) The FL emission spectra of IL-CNRs obtained with different excitation wavelengths. Insets are the IL-CNRs solutions under sunlight (left) and UV light (365 nm).
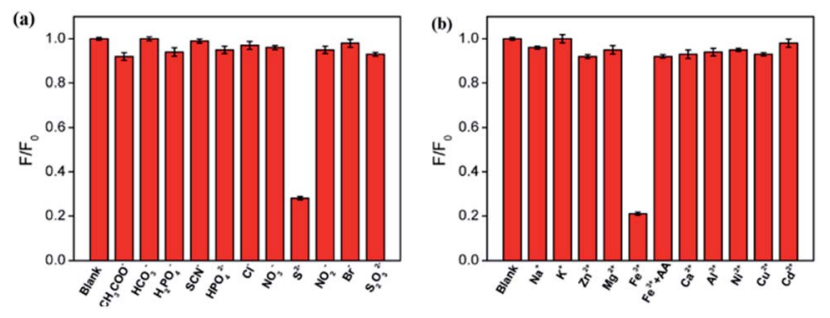

Fig. 5 The relative fluorescent ratio of IL-CNRs in presence of different anion (a) or cation (b). The concentration of ions was $0.3 \mathrm{mM}$.

might be ascribed to the ability of anion exchange originated from IL. ${ }^{32,33,41}$ As fluorescent carbon materials are usually easy to interact with metal cations and cause fluorescence quenching, the selectivity of IL-CNRs towards cations were also investigated. As demonstrated in Fig. 5b, $\mathrm{Fe}^{3+}$ cause fluorescent quenching, while other common cations did not significantly affect the fluorescence intensity of the IL-CNRs. The interactions between IL-CNRs and $\mathrm{Fe}^{3+}$ could be efficiently masked by ascorbic acid (AA). Thus, IL-CNRs have potential for direct and selective detection of $\mathrm{S}^{2-}$ ions.

\subsection{Detection of $S^{2-}$ ions using IL-CNRs as fluorescent probe}

The detection of $\mathrm{S}^{2-}$ ions using IL-CNRs as fluorescent probe was investigated. To obtain the highest sensitivity, the detection conditions including $\mathrm{pH}$ and incubation time were optimized. As illustrated in Fig. S6a (ESI $\dagger$ ), $\mathrm{S}^{2-}$ ions could quench the fluorescence of IL-CNRs very quickly. As the incubation time goes beyond $60 \mathrm{~s}$, the fluorescence quenching reaches a plateau. Obviously, IL-CNRs possesses great potential for fast detection of $\mathrm{S}^{2-}$ ions. Accordingly, the reaction time with IL-CNRs was set as $60 \mathrm{~s}$ in the following experiments. In order to avoid protonation of $\mathrm{S}^{2-}$ ions, fluorescence quenching by $\mathrm{S}^{2-}$ ions was investigated in near neutral solutions. The highest fluorescent

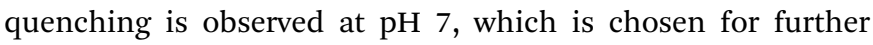
investigated (Fig. S6b in ESI $\dagger$ ).

Owing to the fast and sensitive fluorescence quenching of ILCNRs by $\mathrm{S}^{2-}$ ions (insets in Fig. 6a), the fluorescent detection (a)

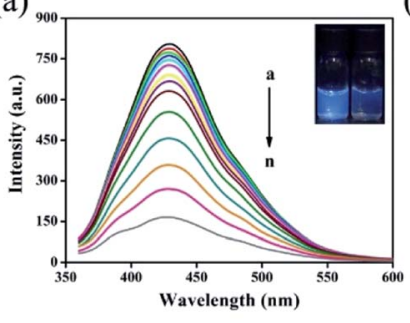

(b)

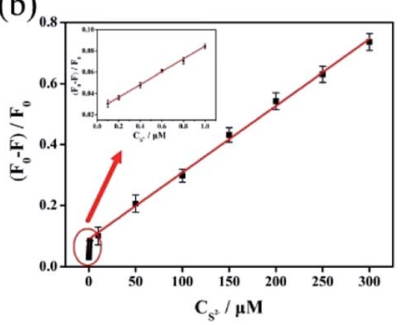

Fig. 6 (a) Fluorescence emission spectra of the IL-CNRs upon addition of various concentrations of $S^{2-}$ (from a to $n$ : $0,0.1,0.2,0.4,0.6$, $0.8,1,10,50,100,150,200,250,300 \mu \mathrm{M})$. Insets are the digital photos of IL-CNRs in the absence (left) and presence (right) of $S^{2-}(100 \mu \mathrm{M})$ under UV light $(365 \mathrm{~nm})$. (b) The linear dependence of the fluorescence quenching ratio $\left(F_{0}-F\right) / F_{0}$ and the concentration of $\mathrm{S}^{2-}$ ions. $F_{0}$ and $F$ were the fluorescence intensity of IL-CNRs in the absence and presence of $\mathrm{S}^{2-}$ ions, respectively. 
was performed under the optimum conditions. As demonstrated in Fig. 6a, $\mathrm{S}^{2-}$ ions caused fluorescence quenching in dose dependent manner. Good linear correlation was found between $\left(F_{0}-F\right) / F_{0}$ and the concentration of $\mathrm{S}^{2-}$ ions from $100 \mathrm{nM}$ to $1 \mu \mathrm{M}$ and 1 to $300 \mu \mathrm{M}$ (Fig. 6b). The limit of detection (LOD) for $\mathrm{S}^{2-}$ ions is as low as $85 \mathrm{nM}$ at a signal-to-noise ratio of 3. Thus, IL-CNRs based method is sensitive enough to detect the maximum allowable level of $\mathrm{S}^{2-}$ ions in drinking water recommended by World Health Organization. ${ }^{42,43}$ As shown in Table S1 (ESI $\dagger$ ), the detection limit obtained with the present method was lower than those obtained by carbon dots (CDs)- $\mathrm{Ag}^{+}{ }^{44}$ covalent linking fluorescein isothiocyanate with branchedpolyethylenimine (PEI-FITC), ${ }^{\mathbf{4 3}}$ triarylimidazole chromophore (TPI-H)-Cu ${ }^{2+}{ }^{45} \mathrm{Au}$ nanoclusters, ${ }^{46}$ lysozyme-stabilized silver nanoclusters (Lys-Ag NCs), ${ }^{47}$ graphene quantum dot (GQDs)$\mathrm{Cu}^{2+},{ }^{48}$ but higher than that obtained using $\mathrm{Cu}$ nanoclusters, ${ }^{49,50}$ Au nanoclusters-Ce(III), ${ }^{51}$ and silver nanoparticles capped with carbon dots (AgNPs-CDs). ${ }^{52}$

\subsection{Real sample analysis}

In order to demonstrate the practical application of the developed fluorescent sensor in detection of $\mathrm{S}^{2-}$ in real samples, tap water samples were analyzed by standard addition method. The recoveries ranges from $\mathbf{9 3 . 7 - 1 0 3 . 8 \%}$ and the relative standard deviations (RSD) is less than $3.0 \%$ (Table $\mathrm{S} 2 \dagger$ ), indicating the potential for the detection of $\mathrm{S}^{2-}$ in complicated real samples.

\section{Conclusions}

Ionic liquid-functionalized carbon nanoribbons (IL-CNRs) were readily synthesized using one-step electrochemical exfoliation of graphite rod in water-IL biphasic system. The hybridization of IL with CNRs not only results in bright fluorescence of CNRs but also endows IL-CNRs with specific interaction with $\mathrm{S}^{2-}$ ions. Taking the advantages of bright photoluminescence and selectivity towards $\mathrm{S}^{2-}$, IL-CNRs are applied for fast, selective and sensitive detection of $\mathrm{S}^{2-}$ ion. In contrast with fluorescent detection of $\mathrm{S}^{2-}$ ion using turn-off-on mode, this IL-CNRs demonstrate its potential for practical use in direct detection of anions.

\section{Conflicts of interest}

There are no conflicts to declare.

\section{Acknowledgements}

The authors gratefully acknowledge the financial support from the National Natural Science Foundation of China (No. 81860512 and 81701721), Guangxi Universities' Young and Middle-aged Teachers Scientific Research Basic Ability Improvement Project (2019KY0139), and the Zhejiang Provincial Natural Science Foundation of China (LY19B050008 and LY20B050007).

\section{References}

1 S. N. Karuk Elmas, F. Ozen, K. Koran, A. O. Gorgulu, G. Sadi, I. Yilmaz and S. Erdemir, Selective and sensitive fluorescent and colorimetric chemosensor for detection of $\mathrm{CO}_{3}{ }^{2-}$ anions in aqueous solution and living cells, Talanta, 2018, 188, 614622.

2 J. M. Jung, J. H. Kang, J. Han, H. Lee, M. H. Lim, K. T. Kim and C. Kim, A novel "off-on" type fluorescent chemosensor for detection of $\mathrm{Zn}^{2+}$ and its zinc complex for "on-off" fluorescent sensing of sulfide in aqueous solution, in vitro and in vivo, Sens. Actuators, B, 2018, 267, 58-69.

3 H. Sammi, D. Kukkar, J. Singh, P. Kukkar, R. Kaur, H. Kaur, M. Rawat, G. Singh and K.-H. Kim, Serendipity in solutionGQDs zeolitic imidazole frameworks nanocomposites for highly sensitive detection of sulfide ions, Sens. Actuators, B, 2018, 255, 3047-3056.

4 Q. Sun, W. Zhang and J. Qian, A ratiometric fluorescence probe for selective detection of sulfite and its application in realistic samples, Talanta, 2017, 162, 107-113.

5 Y. Yao, Q. Sun, Z. Chen, R. Huang, W. Zhang and J. Qian, A mitochondria-targeted near infrared ratiometric fluorescent probe for the detection of sulfite in aqueous and in living cells, Talanta, 2018, 189, 429-436.

$6 \mathrm{H}$. Wu and C. Tong, Nitrogen- and Sulfur-codoped carbon dots for highly selective and sensitive fluorescent detection of $\mathrm{Hg}(2+)$ ions and sulfide in environmental water samples, J. Agric. Food Chem., 2019, 67, 2794-2800.

7 X. Wang, X. Yang, N. Wang, J. Lv, H. Wang, M. Choi and W. Bian, Graphitic carbon nitride quantum dots as an "off-on" fluorescent switch for determination of mercury(II) and sulfide, Microchim. Acta, 2018, 185, 471.

8 C. Wang, J. Sun, H. Mei and F. Gao, Organic semiconductor polymer nanodots as a new kind of off-on fluorescent probe for sulfide, Microchim. Acta, 2016, 184, 445-451.

9 B. Sinduja and S. A. John, Silver nanoparticles capped with carbon dots as a fluorescent probe for the highly sensitive "off-on" sensing of sulfide ions in water, Anal. Bioanal. Chem., 2019, 411, 2597-2605.

10 N. Shahbazi and R. Zare-Dorabei, A novel "off-on" fluorescence nanosensor for sensitive determination of sulfide ions based on carbon quantum dots and gold nanoparticles: Central composite design optimization, Microchem. J., 2019, 145, 996-1002.

11 M. Ramachandran, S. Anandan and M. Ashokkumar, A luminescent on-off probe based calix[4]arene linked through triazole with ruthenium(II) polypyridine complexes to sense copper(II) and sulfide ions, New J. Chem., 2019, 43, 9832-9842.

12 W. Na, Z. Qu, X. Chen and X. Su, A turn-on fluorescent probe for sensitive detection of sulfide anions and ascorbic acid by using sulfanilic acid and glutathione functionalized graphene quantum dots, Sens. Actuators, B, 2018, 256, 48-54.

13 J. Liu, C. Liu and Z. Zhou, A turn-on fluorescent sulfide probe prepared from carbon dots and $\mathrm{MnO}_{2}$ nanosheets, Microchim. Acta, 2019, 186, 281. 
14 X. Chen, S. Yu, L. Yang, J. Wang and C. Jiang, Fluorescence and visual detection of fluoride ions using a photoluminescent graphene oxide paper sensor, Nanoscale, 2016, 8, 13669-13677.

15 Y. Wang, C. Zhang, X. Chen, B. Yang, L. Yang, C. Jiang and Z. Zhang, Ratiometric fluorescent paper sensor utilizing hybrid carbon dots-quantum dots for the visual determination of copper ions, Nanoscale, 2016, 8, 59775984.

16 H. Wang, L. Yang, S. Chu, B. Liu, Q. Zhang, L. Zou, S. Yu and C. Jiang, Semiquantitative visual detection of lead ions with a smartphone via a colorimetric paper-based analytical device, Anal. Chem., 2019, 91, 9292-9299.

17 C. Jiang, B. Liu, M. Han and Z. Zhang, Fluorescent nanomaterials for color-multiplexing test papers toward qualitative/quantitative assays, Small Methods, 2018, 2, 1700379.

18 S. Ge, J. He, C. Ma, J. Liu, F. Xi and X. Dong, One-step synthesis of boron-doped graphene quantum dots for fluorescent sensors and biosensor, Talanta, 2019, 199, 581589.

19 L. Lu, L. Zhou, J. Chen, F. Yan, J. Liu, X. Dong, F. Xi and P. Chen, Nanochannel-confined graphene quantum dots for ultrasensitive electrochemical analysis of complex samples, ACS Nano, 2018, 12, 12673-12681.

20 F. Xi, J. Zhao, C. Shen, J. He, J. Chen, Y. Yan, K. Li, J. Liu and P. Chen, Amphiphilic graphene quantum dots as a new class of surfactants, Carbon, 2019, 153, 127-135.

21 B. C. Chen, H. P. Lin, M. C. Chao, C. Y. Mou and C. Y. Tang, Mesoporous silica platelets with perpendicular nanochannels via a ternary surfactant system, Adv. Mater., 2004, 16, 1657-1661.

22 M. Arjmand, S. Sadeghi, M. Khajehpour and U. Sundararaj, Carbon nanotube/graphene nanoribbon/polyvinylidene fluoride hybrid nanocomposites: rheological and dielectric properties, J. Phys. Chem. C, 2016, 121, 169-181.

23 Y. Yan, J. Gong, J. Chen, Z. Zeng, W. Huang, K. Pu, J. Liu and P. Chen, Recent advances on graphene quantum dots: from chemistry and physics to applications, Adv. Mater., 2019, 31, e1808283.

24 L. Wang, Y. Wang, T. Xu, H. Liao, C. Yao, Y. Liu, Z. Li, Z. Chen, D. Pan, L. Sun and M. Wu, Gram-scale synthesis of single-crystalline graphene quantum dots with superior optical properties, Nat. Commun., 2014, 5, 5357.

25 Y. Yang, J. Zhou, H. Zhang, P. Gai, X. Zhang and J. Chen, Electrochemical evaluation of total antioxidant capacities in fruit juice based on the guanine/graphene nanoribbon/ glassy carbon electrode, Talanta, 2013, 106, 206-211.

26 M. Fukumori, R. R. Pandey, T. Fujiwara, A. TermehYousefi, R. Negishi, Y. Kobayashi, H. Tanaka and T. Ogawa, Diameter dependence of longitudinal unzipping of singlewalled carbon nanotube to obtain graphene nanoribbon, Jpn. J. Appl. Phys., 2017, 56, $06 \mathrm{GG} 12$.

27 S. Sadeghi, M. Arjmand, I. Otero Navas, A. Zehtab Yazdi and U. Sundararaj, Effect of nanofiller geometry on network formation in polymeric nanocomposites: comparison of rheological and electrical properties of multiwalled carbon nanotube and graphene nanoribbon, Macromolecules, 2017, 50, 3954-3967.

28 A. Ananthanarayanan, X. Wang, P. Routh, B. Sana, S. Lim, D.-H. Kim, K.-H. Lim, J. Li and P. Chen, Facile synthesis of graphene quantum dots from 3D graphene and their application for $\mathrm{Fe}^{3+}$ sensing, Adv. Funct. Mater., 2014, 24, 3021-3026.

29 Y. Xu, J. Liu, J. Zhang, X. Zong, X. Jia, D. Li and E. Wang, Chip-based generation of carbon nanodots via electrochemical oxidation of screen printed carbon electrodes and the applications for efficient cell imaging and electrochemiluminescence enhancement, Nanoscale, 2015, 7, 9421-9426.

$30 \mathrm{H}$. Li, L. Chen, H. Wu, H. He and Y. Jin, Ionic liquidfunctionalized fluorescent carbon nanodots and their applications in electrocatalysis, biosensing, and cell imaging, Langmuir, 2014, 30, 15016-15021.

$31 \mathrm{X}$. Li and $\mathrm{Z}$. Zhao, Facile ionic-liquid-assisted electrochemical synthesis of size-controlled carbon quantum dots by tuning applied voltages, RSC Adv., 2014, 4, 57615-57619.

32 B. Wang, W. Tang, H. Lu and Z. Huang, Hydrothermal synthesis of ionic liquid-capped carbon quantum dots with high thermal stability and anion responsiveness, J. Mater. Sci., 2015, 50, 5411-5418.

33 X. Sun, Y. Qian, Y. Jiao, J. Liu, F. Xi and X. Dong, Ionic liquidcapped graphene quantum dots as label-free fluorescent probe for direct detection of ferricyanide, Talanta, 2017, 165, 429-435.

34 M. E. A. Ali, Preparation of graphene nanosheets by electrochemical exfoliation of a graphite-nanoclay composite electrode: application for the adsorption of organic dyes, Colloids Surf., A, 2019, 570, 107-116.

35 D. Chen, F. Wang, Y. Li, W. W. Wang, T. X. Huang, J. F. Li, K. S. Novoselov, Z. Q. Tian and D. Zhan, Programmed electrochemical exfoliation of graphite to high quality graphene, Chem. Commun., 2019, 55, 3379-3382.

36 Y. Fu, G. Gao and J. Zhi, Electrochemical synthesis of multicolor fluorescent $\mathrm{N}$-doped graphene quantum dots as a ferric ion sensor and their application in bioimaging, $J$. Mater. Chem. B, 2019, 7, 1494-1502.

37 X. Li, X. Wang, L. Zhang, S. Lee and H. Dai, Chemically derived, ultrasmooth graphene nanoribbon semiconductors, Science, 2008, 319, 1229-1232.

38 C. Jin, H. Lan, L. Peng, K. Suenaga and S. Iijima, Deriving carbon atomic chains from graphene, Phys. Rev. Lett., 2009, 102, 205501.

39 L. Tapaszto, G. Dobrik, P. Lambin and L. P. Biro, Tailoring the atomic structure of graphene nanoribbons by scanning tunnelling microscope lithography, Nat. Nanotechnol., 2008, 3, 397-401.

40 M. C. Lemme, D. C. Bell, J. R. Williams, L. A. Stern, B. W. H. Baugher, P. Jarillo-Herrero and C. M. Marcus, Etching of graphene devices with a helium ion beam, ACS Nano, 2009, 3, 2674-2676.

41 B. Wang, A. Song, L. Feng, H. Ruan, H. Li, S. Dong and J. Hao, Tunable amphiphilicity and multifunctional 
applications of ionic liquid-modified carbon quantum dots, ACS Appl. Mater. Interfaces, 2015, 7, 6919-6925.

42 J. Chen, Y. Li, K. Lv, W. Zhong, H. Wang, Z. Wu, P. Yi and J. Jiang, Cyclam-functionalized carbon dots sensor for sensitive and selective detection of copper(II) ion and sulfide anion in aqueous media and its imaging in live cells, Sens. Actuators, B, 2016, 224, 298-306.

43 K. Lv, J. Chen, H. Wang, P. Zhang, M. Yu, Y. Long and P. Yi, One-pot fabrication of FRET-based fluorescent probe for detecting copper ion and sulfide anion in $100 \%$ aqueous media, Spectrochim. Acta, Part A, 2017, 177, 63-68.

44 A. Barati, M. Shamsipur and H. Abdollahi, Metal-ionmediated fluorescent carbon dots for indirect detection of sulfide ions, Sens. Actuators, B, 2016, 230, 289-297.

45 Y. Wang, D. Qiu, M. Li, Y. Liu, H. Chen and H. Li, A new "onoff-on" fluorescent probe containing triarylimidazole chromophore to sequentially detect copper and sulfide ions, Spectrochim. Acta, Part A, 2017, 185, 256-262.

46 L. Wang, G. Chen, G. Zeng, J. Liang, H. Dong, M. Yan, Z. Li, Z. Guo, W. Tao and L. Peng, Fluorescent sensing of sulfide ions based on papain-directed gold nanoclusters, New J. Chem., 2015, 39, 9306-9312.
47 H. Sun, D. Lu, M. Xian, C. Dong and S. Shuang, A lysozymestabilized silver nanocluster fluorescent probe for the detection of sulfide ions, Anal. Methods, 2016, 8, 4328-4333.

48 N. Yu, H. Peng, H. Xiong, X. Wu, X. Wang, Y. Li and L. Chen, Graphene quantum dots combined with copper(II) ions as a fluorescent probe for turn-on detection of sulfide ions, Microchim. Acta, 2015, 182, 2139-2146.

49 J. Chen, Y. Li, W. Zhong, Q. Hou, H. Wang, X. Sun, P. Yi and J. Jiang, Novel fluorescent polymeric nanoparticles for highly selective recognition of copper ion and sulfide anion in water, Sens. Actuators, B, 2015, 206, 230-238.

50 Z. Li, S. Guo and C. Lu, A highly selective fluorescent probe for sulphide ions based on aggregation of $\mathrm{Cu}$ nanocluster induced emission enhancement, Analyst, 2015, 140, 27192725.

51 J. Liu, H. Bao, D. Ma and C. Leung, Silver nanoclusters functionalized with $\mathrm{Ce}(\mathrm{III})$ ions are a viable "turn-on-off" fluorescent probe for sulphide, Microchim. Acta, 2019, 186, 16. 52 B. Sinduja and S. Abraham John, Silver nanoparticles capped with carbon dots as a fluorescent probe for the highly sensitive "off-on" sensing of sulfide ions in water, Anal. Bioanal. Chem., 2019, 411, 2597-2605. 